\title{
COPD in the time of COVID-19: an analysis of acute exacerbations and reported behavioural changes in patients with COPD
}

\author{
Hamish McAuley $\mathbb{1}^{1,2}$, Kate Hadley ${ }^{2}$, Omer Elneima ${ }^{1,2}$, \\ Christopher E. Brightling ${ }^{1,2}$, Rachael A. Evans (1) ${ }^{1,2}$, \\ Michael C. Steiner ${ }^{1,2}$ and Neil J. Greening (1) ${ }^{1,2}$
}

Affiliations: 'Dept of Respiratory Sciences, University of Leicester, Leicester, UK. ${ }^{2}$ Institute for Lung Health, NIHR Leicester Biomedical Research Centre, Glenfield Hospital, Leicester, UK.

Correspondence: Neil J. Greening, Dept of Respiratory Sciences, NIHR Leicester BRC - Respiratory Theme, Glenfield Hospital, Groby Road, Leicester, LE3 9QP, UK. E-mail: Neil.greeningaleicester.ac.uk

\section{ABSTRACT}

Introduction: The impact of the severe acute respiratory syndrome coronavirus 2 (SARS-CoV-2) pandemic and associated "lockdown" measures on acute exacerbations of chronic obstructive pulmonary disease (AECOPD) is unknown. We aimed to evaluate the change in AECOPD treatment frequency during the first 6 weeks of lockdown in the UK compared with 2019 and assess changes in self-reported behaviour and wellbeing.

Methods: In this observational study in Leicestershire, UK, patients with COPD under a secondary care clinic were recruited. Exacerbation frequency in the first 6 weeks of COVID-19 lockdown was compared with the same period in 2019 using electronic health records. A telephone survey was used to assess changes in anxiety, inhaler adherence, physical activity and behaviour during the pre-lockdown and lockdown periods compared with normal.

Results: 160 participants were recruited (mean \pm SD age $67.3 \pm 8.1$ years, 88 (55\%) males, mean \pm sD forced expiratory volume in $1 \mathrm{~s} 34 \pm 13 \%$ pred). 140 ( $88 \%$ ) reported at least one AECOPD in the previous year. Significantly more community managed exacerbations were observed in 2020 compared with 2019 (126 versus 99; $\mathrm{p}=0.026$ ). The increase was a result of multiple courses of treatment, with a similar proportion of patients receiving at least one course (34.4\% versus $33.8 \%)$.

Discussion: During lockdown participants reported significantly increased anxiety, adherence to their preventative inhalers and good adherence to shielding advice (all $\mathrm{p}<0.001$ ). A significant reduction in selfreported physical activity and visitors was reported (both $\mathrm{p}<0.001$ ).

Conclusions: Treatment for AECOPD events increased during the first 6 weeks of the SARS-CoV-2 pandemic in the UK compared with 2019. This was associated with increased symptoms of anxiety and significant behavioural change.

$@$ ERSpublications

The \#COVID19 pandemic has changed healthcare provision and access. This study shows a 38\% increase in the number of community treated \#AECOPD in 2020 compared with 2019, alongside increased anxiety and inhaler adherence with reduced physical activity. https://bit.ly/3lV17Q6

Cite this article as: McAuley H, Hadley K, Elneima O, et al. COPD in the time of COVID-19: an analysis of acute exacerbations and reported behavioural changes in patients with COPD. ERJ Open Res 2021; 7: 00718-2020 [https://doi.org/10.1183/23120541.00718-2020].

This article has supplementary material available from openres.ersjournals.com

Data availability: All data requests should be submitted to the corresponding author for consideration. Access to anonymised data may be granted following review.

Received: 2 Oct 2020 | Accepted: 9 Oct 2020

Copyright $\odot$ ERS 2021. This article is open access and distributed under the terms of the Creative Commons Attribution Non-Commercial Licence 4.0. 


\section{Introduction}

Acute exacerbations of chronic obstructive pulmonary disease (AECOPD) are a frequent problem for people with COPD, adversely affecting morbidity and mortality, and are an important cause of unscheduled healthcare contacts, including admission to hospital [1]. The Global Initiative for Chronic Obstructive Lung Disease (GOLD) grades the severity of these events according to treatment requirement, defining moderate events as those needing community provision of oral antibiotics and corticosteroids, and severe events as those requiring hospitalisation [2].

Healthcare provision for people with COPD has been impacted by the severe acute respiratory syndrome coronavirus 2 (SARS-CoV-2) pandemic through the requirement for more distant/remote contact with the healthcare team to reduce the risk of virus transmission [3]. Patients with COPD who present with SARS-CoV-2 infection have a poorer prognosis $[4,5]$, highlighting the need for primary prevention and risk reduction [5]. Additionally, because of the appreciation of a greater risk of morbidity from SARS-CoV-2 infection [6], more stringent social isolation has been recommended for people with COPD during the period of societal "lockdown" that has been implemented in most countries affected by the pandemic. In the UK this has been termed "shielding" and includes advice against leaving home for any reason other than for essential work or shopping, with very limited exceptions.

During this lockdown period healthcare professionals providing care for people with COPD have reported lower than expected presentation rates for AECOPD in both community and acute hospital settings $[7,8]$. However, it is unclear whether this is due to a genuine reduction in AECOPD rates (potentially due to lower respiratory viral transmission [9] and/or atmospheric pollution) or due to higher thresholds for patients reporting to healthcare services because of fearfulness about contracting SARS-CoV-2 in healthcare environments, particularly hospitals. In addition there is limited understanding of the impact of enhanced shielding on psychological wellbeing and physical activity in people with pre-existing respiratory disease such as COPD [10, 11]. Having a chronic condition such as COPD does not appear to increase the likelihood of SARS-CoV-2 infection [12], although it does convey increased risk of hospitalisation and death [6].

First, in this observational study we recorded the change in moderate and severe AECOPD treatment frequency (assessed objectively through prescription records or requirement for hospitalisation) during the first 6 weeks of societal lockdown in the UK compared with the equivalent period 12 months previously. Second, we assessed self-reported behaviour change during the pre-lockdown and lockdown periods by telephone interview in order to explore potential reasons for any observed changes in AECOPD treatment frequency

\section{Methods}

\section{Study design}

We compared rates of treatment for exacerbations of COPD managed in the community and hospital setting in a single centre (Glenfield Hospital, Leicester, UK) between the first 6-week period of the SARS-CoV-2 lockdown in England (March 15, 2020 to April 30, 2020) with the same 6-week period in the previous year (March 15, 2019 to April 30, 2019).

Participants were prospectively recruited between June 2, 2020 and July 8, 2020, and provided informed consent. All recruitment and interview calls were made either by experienced respiratory research nurses or clinicians. Ethics approval was granted by the London-Brent Research Ethics Committee (20/HRA/2510).

Electronic community prescription records were used to record community exacerbation events and electronic hospital records similarly for hospital exacerbations. The terms community and hospital exacerbation, rather than moderate or severe [2], are used in this study due to the known change in hospital admission criteria during the early peak of the SARS-CoV-2 pandemic in England when hospital bed capacity was considered of critical importance. Community managed exacerbations were defined as those resulting in a prescription for oral corticosteroids and/or antibiotics but without hospital admission. Only prescriptions for antibiotics that would typically be used to treat AECOPD events in our region were included. Hospitalised exacerbations were defined as admissions to hospital with a recorded discharge diagnosis of AECOPD.

As an additional analysis, a scripted telephone survey was conducted to explore potential reasons for differences in exacerbation risk. Participants were asked to compare behavioural and emotional changes with their baseline "normal" state as a reference. Participants were asked to compare two discrete periods: 1) pre-lockdown, defined as the 2 weeks prior to lockdown (March 1, 2020 to March 14, 2020) when participants were likely to be more aware of the threat of SARS-CoV-2 but restrictions had not yet been put in place, and 2) the lockdown itself (March 15, 2020 to April 20, 2020). Self-reported behaviour included medication adherence to their regular prescribed inhaled therapy, anxiety, change in activity 
levels and social behaviour (self-isolation, shielding, visitors to the home and arrangements for shopping). Answers were captured with either a binary response (yes/no) or on a five-point Likert scale (see supplementary material for full details of the questionnaire used).

\section{Study population}

Participants were eligible if they had a confirmed diagnosis of COPD, under a specialist COPD clinic (Complex COPD clinic, Leicester, UK [13]) and able to provide informed verbal consent via English language telephone consultation. The specialist clinic accepts any patient with COPD with ongoing symptoms (e.g. admission to hospital for AECOPD, modified Medical Research Council Dyspnoea scale grade $>2$, continued smoking, low body mass index, potentially lung volume reduction candidate or established respiratory failure). All patients had confirmed airflow obstruction by GOLD criteria [2]. Patients were contacted sequentially from the research database held in our centre of 258 patients alive at the start of the study who had previously consented to be contacted for research until this list was exhausted, with a total of three attempts to contact participants who did not answer. The telephone call was made by either a nurse or doctor and participants gave informed consent verbally, which was documented by the investigator due to the remote nature of the consultation.

Electronic general practice and hospital healthcare records were used to capture new prescriptions for oral antibiotics or corticosteroids during the periods of interest, hospital admissions, as well as baseline characteristics, including latest spirometry. All spirometry had been performed at their previous clinic visit to Glenfield Hospital according to European Respiratory Society/American Thoracic Society standards [14].

\section{Statistical analysis}

Baseline data were described as mean with standard deviation or number (percentage) as appropriate. Paired data were compared using the paired t-test or signed-rank test for parametric data or nonparametric data, respectively. Categorical data were compared using the Chi-squared test. Statistical analysis was performed using Stata version 16 (StataCorp, College Station, TX, USA).

From previous data from our COPD clinic we anticipated 0.8 exacerbations per patient in the observation period with a SD of 0.9. To detect a 25\% difference in exacerbations within patients between 2019 and 2020, 160 participants would be required ( $\alpha=0.05$, power $80 \%)$.

\section{Results}

160 participants were recruited with baseline characteristics outlined in table 1.140 (88\%) participants reported at least one exacerbation in the previous year and the majority $(103(64 \%))$ reported at least two exacerbations. 149 (93\%) participants were prescribed triple inhaled therapy and 138 (86\%) were classed as GOLD stage 3 or 4 airflow obstruction.

\section{Number of exacerbations}

In the first 6 weeks of the lockdown period (March 15, 2020 to April 30, 2020) there were significantly more total exacerbations events compared with the same period in 2019 (126 versus 99 events; $\mathrm{p}=0.026$ ). The number of exacerbations per patient is shown in figure 1a. Patients receiving at least one course of treatment in 2020 were more likely to receive multiple courses than in 2019 (table 2), while overall there was a similar proportion of patients who received at least one course of treatment $(34.4 \%$ in 2020 versus $33.8 \%$ in 2019) (supplementary figure S1).

Unsurprisingly, community managed events comprised the majority of exacerbations (209 out of 225 (93\%)). For community exacerbations alone, 121 events were noted in 2020 compared with 88 events in 2019 ( $p=0.004$ ) (figure 1b). There were five hospitalisations $(n=5)$ due to AECOPD during the lockdown period in 2020 compared with 10 hospitalisations $(n=9)$ in the same period in 2019 (figure 1c).

\section{Medication adherence}

In the 2 weeks prior to lockdown 131 (83\%) participants reported using their maintenance inhalers with the same frequency as they would during their stable state. 23 (14\%) participants reported increased use and four (2.5\%) reported use less frequently than normal. During the lockdown period 42 (26\%) participants reported increased use, 113 (71\%) reported the same frequency of use and four $(2.5 \%)$ reported using their regular inhaler less frequently than baseline $(p<0.001)$ (figure $2 a)$.

\section{Anxiety}

45 (28\%) participants reported having more anxiety about their COPD than normal during the pre-lockdown period compared with baseline, of which 42 out of 45 (93\%) reported anxiety as a "little more" than baseline and three out of $45(7 \%)$ as "much more" than baseline. During the lockdown period 


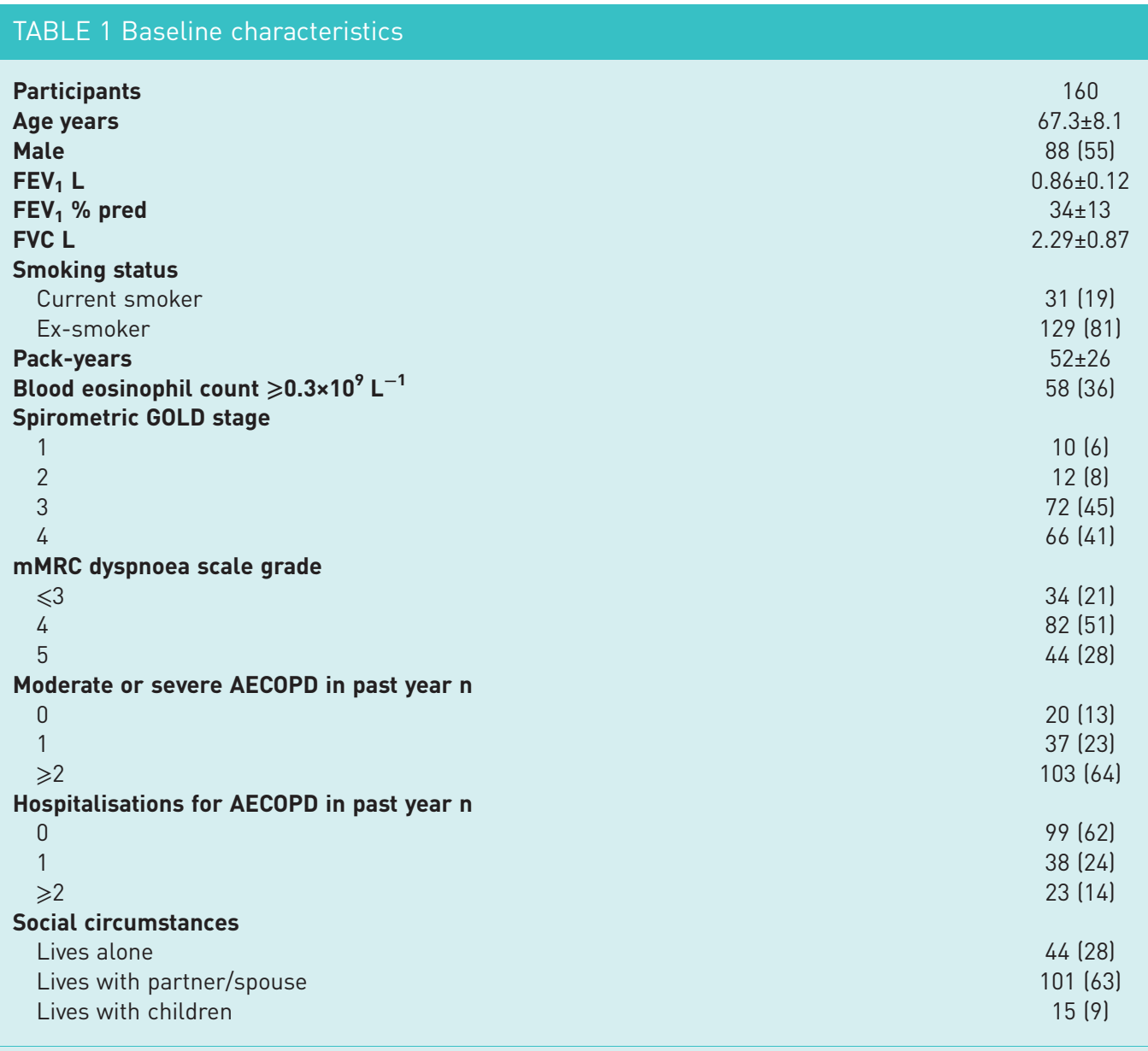

Data are presented as $\mathrm{n}$, mean $\pm \mathrm{SD}$ or $\mathrm{n}(\%) . \mathrm{FEV}_{1}$ : forced expiratory volume in $1 \mathrm{~s}$; FVC: forced vital capacity; GOLD: Global Initiative for Chronic Obstructive Lung Disease; mMRC: modified Medical Research Council; AECOPD: acute exacerbations of chronic obstructive pulmonary disease.

$92(58 \%)$ participants reported increased anxiety compared with normal $(\mathrm{p}<0.001)$, of which 48 out of 92 (52\%) were a "little more" anxious and 42 out of 92 (48\%) were "much more" anxious (figure 2b).

Participants were also asked if they would avoid coming to hospital as an emergency during the pre-lockdown and lockdown periods due to fear of COVID-19. 64 (40\%) participants reported they would have avoided doing so during the pre-lockdown and 90 (56\%) reported they would avoid emergency hospital attendance during the lockdown period $(\mathrm{p}<0.001)$.
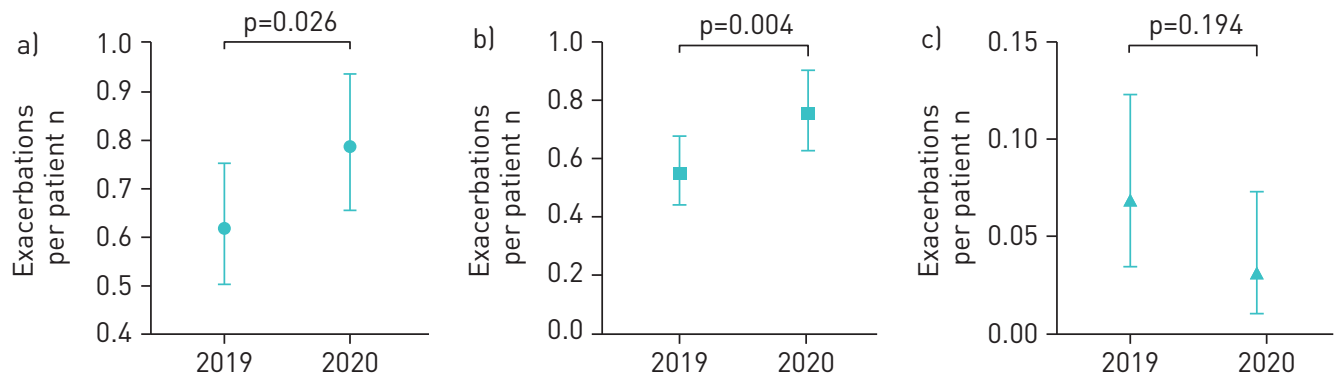

FIGURE 1 Number of exacerbations per patient between March 15 and April 30 in 2019 and 2020: a) all community and hospitalised managed exacerbations, b) community managed exacerbations only, and c) hospitalised managed exacerbations only. Data are presented as mean with $95 \%$ confidence interval. 


TABLE 2 Number of participants suffering exacerbation events (prescriptions for antibiotics or
corticosteroids or both in the community or admissions to hospital) grouped by the number of
events recorded during the 6-week reference period in each year

\section{Physical activity and exercise}

83 (54\%) participants reported physical activity was unchanged compared with normal during the pre-lockdown period, with $63(40 \%)$ reporting reduced activity levels and $11(7 \%)$ reporting increased activity levels. This contrasted sharply to the lockdown period where only 26 (16\%) participants reported maintaining the same level of activity as normal, while $52(32.5 \%)$ reported doing slightly less and 78 (49\%) reported doing a lot less physical activity than normal, implying a significant decrease in activity levels $(\mathrm{p}<0.001)$. Only four $(2 \%)$ participants reported increased physical activity levels (figure $2 c)$. When asked about participation in a home exercise programme, 50 (31\%) and 56 (35\%) patients reported participating in a home exercise programme during the pre-lockdown and lockdown periods, respectively.

\section{Shopping behaviour}

Participants were asked about shopping behaviour during the pre-lockdown and lockdown periods, with a significant change being noted; during the pre-lockdown period 89 (55.6\%) participants reported going shopping themselves, while $33(20.6 \%)$ reported that this was performed by someone who lives in the

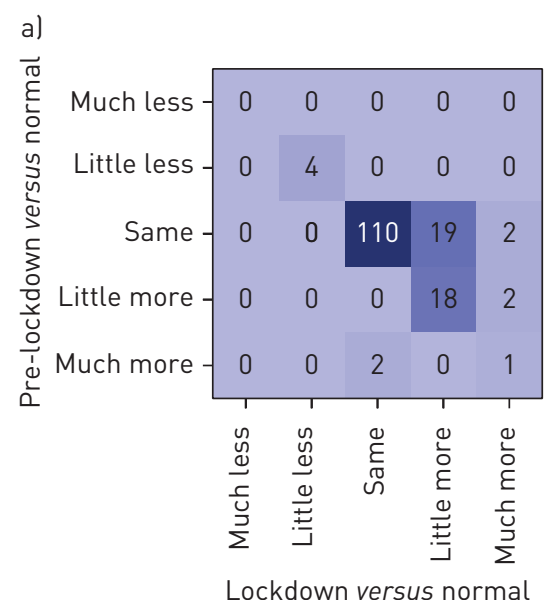

Lockdown versus normal

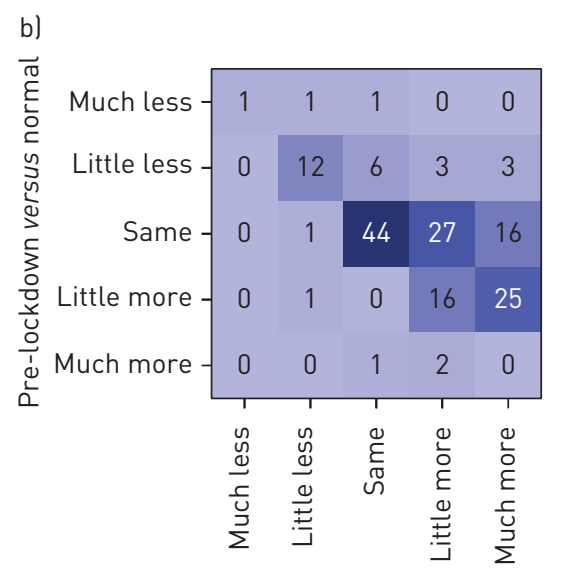

Lockdown versus normal

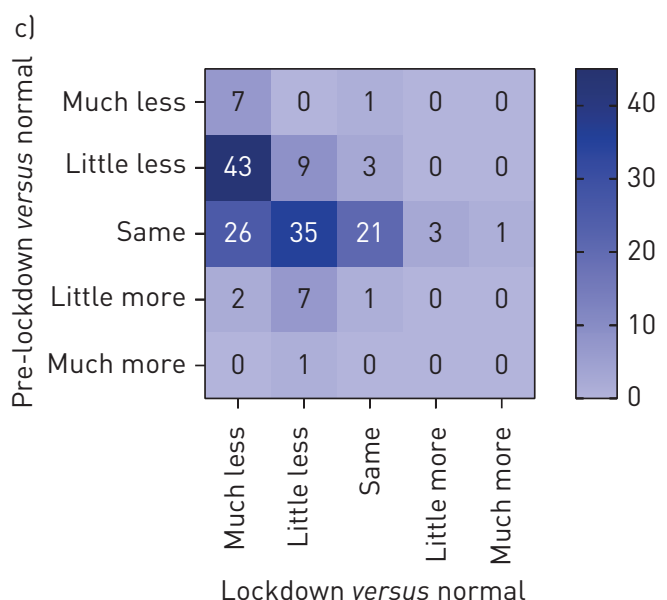

Lockdown versus normal

FIGURE 2 Changes in self-reported behaviour in the 2 weeks pre-lockdown and during the first 6 weeks of lockdown compared with normal baseline for a) regular inhaler use, b) anxiety and c) physical activity. Change between pre-lockdown and lockdown for all groups p<0.001. 
house with them and 38 (23.8\%) reported it being completed by someone who does not live with them or being delivered to them. In contrast to this, during the lockdown period only 11 (6.9\%) participants reported that they still did their own shopping, with 37 (23.1\%) having this task completed by someone living in their home and $112(70 \%)$ reporting that it was done by someone who does not live in their home or delivered (pre-lockdown to lockdown; $\mathrm{p}<0.001$ ).

\section{Shielding and visitors}

In the pre-lockdown period $142(88.8 \%)$ participants reported continuing normal behaviour, with only 16 (10\%) shielding. Once lockdown started only seven (4.4\%) participants reported continuing normal behaviour, while $127(79.9 \%)$ reported that they were shielding $(\mathrm{p}<0.001)$.

During the pre-lockdown period 146 (91.3\%) participants reported that they had visitors to their home compared with 31 (19.4\%) during lockdown $(\mathrm{p}<0.001)$.

\section{Discussion}

In this observational study a $38 \%$ increase in community managed exacerbation events during the COVID-19 lockdown in 2020 was seen compared with the same 6-week period in 2019, as measured by primary care prescription records. The number of patients suffering an exacerbation was unchanged. Self-reported anxiety and inhaler adherence increased, whereas physical activity was lower initially during the pre-lockdown period but most pronounced during lockdown.

Severe exacerbations, as measured by hospital admissions, were seen within the cohort and represented 6\% of all exacerbations. We observed a 50\% decrease in hospital managed AECOPD events during the COVID-19 lockdown compared with the same period in 2019, although our study was insufficiently powered. A recent larger study, comparing hospital events rather than individual patients, confirms our observations with a similar reduction in AECOPD admission rates [8]. This may represent an effect of the increased use of rescue medications in the community resulting in reduced need for hospital admissions, although other factors are also likely to have played a role.

This is the first report of the impact of the SARS-CoV-2 pandemic (and consequent societal lockdown) on objectively measured AECOPD rates. Our findings contrast with reports of reduced AECOPD events during lockdown from physicians and COPD nursing teams [7]. Interestingly, we did not observe an increase in the proportion of patients requiring rescue medication, but did observe an increase in the number of multiple courses. Possible explanations for these findings may result from either biological or behavioural differences. Patients who would normally have been admitted to hospital with an exacerbation may have been managed in the community during the pandemic because of a combination of fearfulness on the part of the patient about transmission risk in hospital and a desire on the part of healthcare teams to reserve hospital bed capacity to manage patients suffering with COVID-19 pneumonia. This behavioural explanation appears plausible, particularly as there was increased access to healthcare services via telephone consultations and reduced physical access to clinicians [15]. National guidance, updated in 2018, recommends an "action plan" which includes oral corticosteroids and antibiotics to be self-administered in the event of an AECOPD [16]. Increased concerns on the part of clinicians about the risks of hospitalisation in a patient population perceived to be at greater risk from SARS-CoV-2 might have lowered thresholds for prescribing action plans. Patient concern that access to primary or secondary healthcare teams and pharmacies might be restricted may also have resulted in stockpiling behaviour during the pandemic [17], with patients potentially requesting multiple "rescue packs" to store in case they were unable to obtain these later. During remote telephone consultations clinicians may have felt unable to assess the severity of patients' symptoms, resulting in a reduced threshold for prescribing acute rescue therapies. These behavioural aspects may provide cautions beyond the current pandemic about how future digital health services and clinics are arranged and incentivised.

It is possible that the biological triggers for exacerbation events were reduced for some patients because of reduced respiratory virus transmission and air pollution during lockdown. Our study did not explore the underlying mechanisms of each exacerbation, which may be altered during the SARS-CoV-2 pandemic. It is possible that events could be driven by noninflammatory causes, termed "pauci-inflammatory" [18], which are less responsive to oral antibiotics or corticosteroids [19]. We observed that the majority of participants reported increasing anxiety about their COPD, particularly during the lockdown period. This would support a view that the observed increase in exacerbations may be underpinned by behavioural change and concerns around COPD and emergency healthcare. It also highlights the need for potential psychological support in a vulnerable population, where anxiety and depression are common [20].

In addition, it is clear from our data that adherence to shielding advice was widespread, likely reflecting a shared concern among patients about risks from COVID-19. Likewise, we observed an increase in self-reported inhaler compliance, suggesting greater health concerns and vigilance. 
We also observed a greater dependence on others for day-to-day activities such as shopping and an overall reduction in physical activity among this cohort of patients with COPD that contrasts with that reported among the general population during the lockdown period [21]. Previous studies have demonstrated the association of reduced physical activity levels and AECOPD readmission risk [22]; while this study did not directly assess this effect, it raises the additional possibility that exacerbation events increased because of increased breathlessness and reduced resilience due to deconditioning and sarcopenia [23, 24]. The longer-term consequences of such altered activity behaviours remains to be seen, but is of significant concern given the difficulty in providing timely and effective pulmonary rehabilitation in the context of the pandemic [25].

At the time of writing, we are approaching winter in the northern hemisphere and no SARS-CoV-2 vaccine has yet been demonstrated to be safe and effective [26]; exacerbations of COPD are likely to increase with this season and result in increased hospitalisation. This study is a timely reminder that increased understanding of community prescribing practice and patient behaviour are important, and may reveal effective tools in reducing morbidity and mortality in this group. First, patients with COPD are going to require ongoing support and treatment, even if they are less likely to present to specialist or hospital services. Previous evidence has shown that pandemic influenza poses a significant risk to patients with COPD, with the consequence that viral pandemics such as SARS-CoV-2 are likely to pose a similar risk [27]. Developing robust and accessible systems to acutely review patients with COPD remotely to guide them in their use of rescue and preventer medication may reduce symptom burden, hospital admissions, and unnecessary courses of potentially harmful oral corticosteroids and antibiotics. It is less likely that the increased number of moderate exacerbations recorded from our prescription data represents an increase in airway inflammation, but rather a composite of increased anxiety and caution with the aim of preventing hospital admissions and the consequence that other, nonpharmacological interventions may have been effective in managing these events [27].

The conclusions drawn from this study are limited by both the relatively small sample size and the severity of the COPD seen in the cohort recruited. Although the dataset of 160 patients provided adequate power for statistically significant differences in community managed exacerbation and behavioural changes, it was not sufficiently large to detect changes in hospitalised events, which would be better evaluated using larger datasets. In addition to this, the cohort had established COPD, under a specialist secondary care clinic, so results may not be applicable to those with milder disease and less frequent exacerbations. Adding further selection bias, patients recruited needed to be alive during the period of recruitment in May and June 2020, meaning that there may be survivor bias compared with those that died in 2019 and during the peak of the pandemic. Our use of a survey to assess associated factors and explore possible causes for patterns seen was notably limited by recollection bias, with questionnaire calls taking place up to 7 weeks after the end of the period of interest, and by our use of nonvalidated questionnaires.

In summary, this study revealed an increase in treatment for community treated AECOPD events among patients with severe COPD during the SARS-CoV-2 lockdown (March 15, 2020 to April 20, 2020) in the UK. This finding was unexpected but may be explained by factors such as anxiety, which was increased in our patient cohort. Significant behaviour changes including reduced physical activity, adherence to shielding advice and increased inhaler compliance.

Acknowledgements: We wish to acknowledge the work of the following members of the NIHR Leicester Biomedical Research Centre Respiratory team and University Hospitals of Leicester (Leicester, UK): Sarah Parker, Jo Finch, Sarah Glover, Tracy Thorn, Bo Zhao and Adrian Manise.

Conflict of interest: H. McAuley has nothing to disclose. K. Hadley has nothing to disclose. O. Elneima has nothing to disclose. C.E. Brightling has nothing to disclose. R.A. Evans reports Clinical Scientist Fellowship CS-2016-16-020 from the NIHR during the conduct of the study. M.C. Steiner has nothing to disclose. N.J. Greening reports Post-Doctoral Fellowship PDF-2017-10-052 from the NIHR during the conduct of the study.

Support statement: The research was supported by the NIHR Leicester Biomedical Research Centre - Respiratory Theme. N.J. Greening is funded by a NIHR Post-Doctoral Fellowship (PDF-2017-10-052). R.A. Evans is funded by a NIHR Clinician Scientist Fellowship (CS-2016-16-020). The views expressed are those of the author(s) and not necessarily those of the National Health Service and NIHR or the Dept of Health. Funding information for this article has been deposited with the Crossref Funder Registry.

\section{References}

1 Suissa S, Dell'Aniello S, Ernst P. Long-term natural history of chronic obstructive pulmonary disease: severe exacerbations and mortality. Thorax 2012; 67: 957-963.

2 Global Initiative for Chronic Obstructive Lung Disease. Global Strategy for the Diagnosis, Management and Prevention of Chronic Obstructive Pulmonary Disease. 2019. www.goldcopd.org/wp-content/uploads/2019/12/ GOLD-2020-FINAL-ver1.2-03Dec19_WMV.pdf Date last accessed: October 23, 2020. 
3 National Institute for Health Care and Excellence. COVID-19 Rapid Guideline: Community-based Care of Patients with Chronic Obstructive Pulmonary Disease (COPD). NICE Guideline 168. 2020. www.nice.org.uk/ guidance/ng168 Date last accessed: September 16, 2020.

4 Williamson EJ, Walker AJ, Bhaskaran K, et al. Factors associated with COVID-19-related death using OpenSAFELY. Nature 2020; 584: 430-436.

5 Leung JM, Niikura M, Yang CWT, et al. COVID-19 and COPD. Eur Respir J 2020; 56: 2002108.

6 Alqahtani JS, Oyelade T, Aldhahir AM, et al. Prevalence, severity and mortality associated with COPD and smoking in patients with COVID-19: a rapid systematic review and meta-analysis. PLoS One 2020; 15: e0233147.

7 Hurst J. Twitter post We continue to see very few \#COPD exacerbations, and not just because people aren't coming to hospital ... 2020. https://twitter.com/ProfHurst/status/1249980803624435714 Date last accessed: November 29, 2020.

8 Berghaus TM, Karschnia P, Haberl S, et al. Disproportionate decline in admissions for exacerbated COPD during the COVID-19 pandemic. Respir Med 2020; in press [http://dx.doi.org/10.1016/j.rmed.2020.106120]

9 Mathioudakis AG, Janssens W, Sivapalan P, et al. Acute exacerbations of chronic obstructive pulmonary disease: in search of diagnostic biomarkers and treatable traits. Thorax 2020; 75: 520-527.

10 Simons SO, Hurst JR, Miravitlles M, et al. Caring for patients with COPD and COVID-19: a viewpoint to spark discussion. Thorax 2020; 75: 1035-1039.

11 Pfefferbaum B, North CS. Mental health and the Covid-19 pandemic. N Engl J Med 2020; 383: 510-512.

12 Guan W-J, Liang W-H, Zhao Y, et al. Comorbidity and its impact on 1590 patients with COVID-19 in China: a nationwide analysis. Eur Respir J 2020; 55: 2000547.

13 Steiner MC, Evans RA, Greening NJ, et al. Comprehensive respiratory assessment in advanced COPD: a 'campus to clinic' translational framework. Thorax 2015; 75: 805-808.

14 Miller MR, Hankinson J, Brusasco V, et al. Standardisation of spirometry. Eur Respir J 2005; 26: 319-338.

15 Budd J, Miller BS, Manning EM, et al. Digital technologies in the public-health response to COVID-19. Nat Med 2020; 26: 1183-1192.

16 Hopkinson NS, Molyneux A, Pink J, et al. Chronic obstructive pulmonary disease: diagnosis and management: summary of updated NICE guidance. BMJ 2019; 366: 14486.

17 Hall MC, Prayag G, Fieger P, et al. Beyond panic buying: consumption displacement and COVID-19. J Serv Manag 2020; in press [https://doi.org/10.1108/JOSM-05-2020-0151].

18 Bafadhel M, McKenna S, Terry S, et al. Acute exacerbations of chronic obstructive pulmonary disease identification of biologic clusters and their biomarkers. Am J Respir Crit Care Med 2011; 184: 662-671.

19 Pooler A, Beech R. Examining the relationship between anxiety and depression and exacerbations of COPD which result in hospital admission: a systematic review. Int I Chron Obstruct Pulmon Dis 2014; 9: 315-330.

20 Coventry PA, Hind D. Comprehensive pulmonary rehabilitation for anxiety and depression in adults with chronic obstructive pulmonary disease: systematic review and meta-analysis. J Psychosom Res 2007; 63: 551-565.

21 Ding D, del Pozo Cruz B, Green MA, et al. Is the COVID-19 lockdown nudging people to be more active: a big data analysis. Br J Sports Med 2020; 54: 1183-1184.

22 Garcia-Aymerich J, Farrero E, Félez MA, et al. Risk factors of readmission to hospital for a COPD exacerbation: a prospective study. Thorax 2003; 58: 100-105.

23 Greening NJ, Harvey-Dunstan TC, Chaplin EJ, et al. Bedside assessment of quadriceps muscle using ultrasound following admission for acute exacerbations of chronic respiratory disease. Am J Respir Crit Care Med 2015; 192: $810-816$

24 Kon SS, Jones SE, Schofield SJ, et al. Gait speed and readmission following hospitalisation for acute exacerbations of COPD: a prospective study. Thorax 2015; 70: 1131-1137.

25 Houchen-Wolloff L, Steiner MC. Pulmonary rehabilitation at a time of social distancing: prime time for tele-rehabilitation? Thorax 2020; 75: 446-447.

26 Bar-Zeev N, Inglesby T. COVID-19 vaccines: early success and remaining challenges. Lancet 2020; 396: 868-869.

27 Hewitt R, Farne H, Ritchie A, et al. The role of viral infections in exacerbations of chronic obstructive pulmonary disease and asthma. Ther Adv Respir Dis 2016; 10: 158-174. 